Vol. 131: 1-11, 2018

\title{
Retrospective pathological survey of pulmonary disease in free-ranging Amazon river dolphin Inia geoffrensis and tucuxi Sotalia fluviatilis
}

\author{
Thaís Carneiro S. Rodrigues ${ }^{1,2, *}$, Josué Díaz-Delgado ${ }^{3}$, José Luiz Catão-Dias ${ }^{3}$, \\ Júlia da Luz Carvalho ${ }^{2}$, Miriam Marmontel ${ }^{2}$ \\ ${ }^{1}$ PhD Program on Veterinary Sciences, Federal University of Uberlândia (UFU). Uberlândia, MG 38400-902, Brazil \\ ${ }^{2}$ Mamirauá Institute for Sustainable Development (IDSM), Tefé, AM 69553-225, Brazil \\ ${ }^{3}$ Laboratory of Wildlife Comparative Pathology, Department of Pathology, \\ School of Veterinary Medicine and Animal Science, University of São Paulo, São Paulo, SP, 05508-270, Brazil
}

\begin{abstract}
The Amazon river dolphin Inia geoffrensis and tucuxi Sotalia fluviatilis are classified as Data Deficient species. Despite very limited knowledge on health and disease aspects of these species, the main threats to their conservation include incidental mortality in fishing gear, population fragmentation, habitat loss and environmental pollution. It is also suggested that underlying diseases may contribute to their mortality rates. Herein, we retrospectively describe gross and microscopic pulmonary lesions in free-ranging $I$. geoffrensis $(\mathrm{n}=24)$ and $S$. fluviatilis $(\mathrm{n}=28)$ found dead. Nearly $85 \%$ of the examined animals presented some kind of primary lung disease, wherein the main etiological diagnoses were verminous pneumonia by Halocercus brasiliensis $(25 \%)$, bacterial pneumonia $(25 \%)$ and a single case of meconium aspiration syndrome $(1.9 \%)$. An etiology was not determined in $36.5 \%$ (19/52) of animals. These results indicate a high incidence of pulmonary pathology in these species, raising concerns about population impacts and potential zoonotic implications in some instances. These data may provide a scientific basis for future medical and conservation efforts focused on Amazonian dolphins.
\end{abstract}

KEY WORDS: Cetacean $\cdot$ Halocercus brasiliensis $\cdot$ Lung $\cdot$ Odontocete $\cdot$ Pathology $\cdot$ Pneumonia River dolphin $\cdot$ Streptococcus iniae

\section{INTRODUCTION}

The Amazon river dolphin Inia geoffrensis is the world's largest freshwater river dolphin and is endemic to the Amazon and Orinoco basins, occurring in Brazil, Colombia, Ecuador, Peru and Venezuela (Reeves et al. 2011). In contrast, the tucuxi Sotalia fluviatilis is the world's smallest and only exclusively freshwater delphinid. This species is largely sympatric with I. geoffrensis, being endemic to the Amazon basin in Brazil, Colombia, Ecuador and Peru (Secchi 2012). Both dolphin species are regarded as indicators of the Amazon's aquatic ecosystem quality and play important roles in keeping fish populations healthy and balanced, removing ill individuals and controlling the most abundant species (ICMBio 2011).

Due to the limited knowledge on ecology, population and threats, I. geoffrensis and S. fluviatilis are considered Data Deficient by the International Union for Conservation of Nature (IUCN) (Reeves et al. 2011, Secchi 2012). In Brazil, these species were classified as Near Threatened in 2003 (ICMBio 2011), but the Amazon river dolphin's status was uplisted to Endangered at the end of 2014 (Brazil 2014) due to its 
deliberate killing for use as fish bait (Iriarte \& Marmontel 2013a). Major known threats include incidental mortality in fishing gear, population fragmentation and isolation, habitat loss and environmental pollution (Reeves et al. 2011, Secchi 2012).

In addition to these main threats, it is suggested that underlying pre-existing diseases may contribute towards death. These odontocete species are susceptible to a number of natural pathological processes, including zoonotic agents, e.g. Streptococcus iniae, that could contribute to population decline (Bonar \& Wagner 2003, Bonar et al. 2007). A retrospective pathological survey on worldwide captive $I$. geoffrensis reported pneumonia as the most common finding, affecting $42 \%$ of the necropsied animals (Bonar et al. 2007). In fact, pneumonia and other respiratory diseases have been recognized as important causes of death in both captive and free-ranging cetaceans (Dunn \& Buck 2001, Venn-Watson et al. 2012). None- theless, very limited and fragmented information regarding the pathology of free-ranging I. geoffrensis and S. fluviatilis is available in the literature.

Aiming to partially fill in this gap of knowledge, we retrospectively described the gross and microscopic lesions in a sample of these dolphin species from the mid-Solimões River. These results may provide a scientific basis for future medical and conservation efforts focused on Amazonian dolphins.

\section{MATERIALS AND METHODS}

From 1995 to 2016, carcasses of free-living Inia geoffrensis and Sotalia fluviatilis were opportunistically collected in the areas of Amanã and Mamirauá Sustainable Development Reserves (ASDR and MSDR) and Lake Tefé, Amazonas state, Brazil (Fig. 1). The studied area is included in the Amazon-

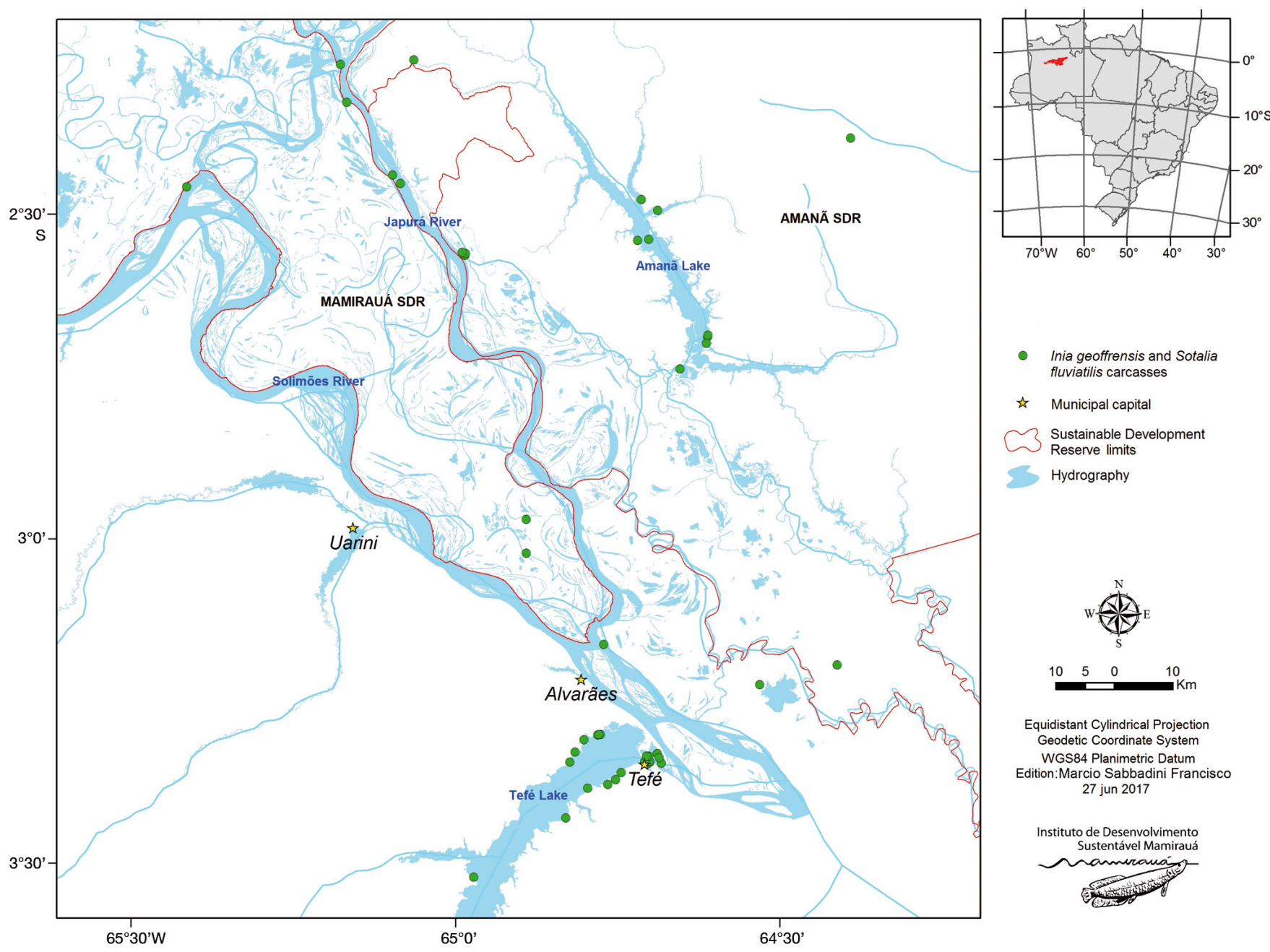

Fig. 1. Geographical distribution of Amazon river dolphin Inia geoffrensis and tucuxi Sotalia fluviatilis carcasses collected in the mid-Solimões River, Brazil (1995-2016) 
ian várzea ecosystem, an annually inundated seasonal floodplain forest located in the Amazon biome, between the Negro, Japurá and Solimões river basins, in the mid-Solimões River. Temperature and rainfall are high all year long. Both riverine dolphin species occur extensively throughout the studied area, although population estimates are not available. Human populations of the ASDR and the MSDR are distributed in small groups and riverside communities, whose main subsistence activities are agriculture, extractivism, fishing and hunting (www. mamiraua.org.br/pt-br/reservas/). Fishing is also an important activity in Lake Tefé, a non-protected lake located adjacent to the city of Tefé, an important Amazonian urban center (Vianna et al. 2010).

The dolphin carcasses were subjected to partial $(\mathrm{n}=27)$ or complete $(\mathrm{n}=25)$ standardized necropsies. Age class was determined based on total body length (Best \& da Silva 1984). Where possible due to the carcass condition, the nutritional status of each animal was established morphologically with reference to anatomical parameters such as the osseous prominence of the spinous and transverse vertebral processes and ribs, the mass of the epaxial musculature (longissimus dorsi, multifidus and spinalis), and the absence or limited presence of subcutaneous and cavitary fat deposits. These parameters allowed us to classify their nutritional status as good or emaciated. The preservation status (decomposition code) of the carcasses was classified as fresh, moderate autolysis, or advanced autolysis. Representative samples of the right and left lungs were collected and fixed in $10 \%$ neutral buffered formalin. The tissue samples were processed as routine and embedded in paraffin, and $5 \mu \mathrm{m}$ sections were stained with hematoxylin and eosin (H\&E) for light microscopic analysis. Selected tissue sections were also stained with Gram/Twort and Grocott stains, for bacteria and fungi, respectively. Parasitological identification relied on macroscopic and histologic features in tissue sections (Delyamure 1955, Anderson et al. 2009).

\section{RESULTS}

\section{Epidemiology}

We examined 24 Inia geoffrensis and 28 Sotalia fluviatilis. Age categories represented were adults $(50 \% ; 26 / 52)$, juveniles $(30.7 \% ; 16 / 52)$ and neonates $(9.6 \% ; 5 / 52)$. The examined specimens included 22 females $(42.3 \%)$ and 22 males $(42.3 \%)$. Age and sex could not be determined in $5(9.6 \%)$ and 8 cases
$(15.4 \%)$, respectively. Although the nutritional status was not determined in most cases $(80.8 \%$; 42/52), 8 carcasses were deemed good $(15.4 \%)$ and 2 were emaciated $(3.8 \%)$. Seventeen $(32.7 \%)$ carcasses were fresh, $13(25 \%)$ were in moderate autolysis, 6 $(11.5 \%)$ were in advanced autolysis and in 16 $(30.8 \%)$ cases the decomposition state was not determined. General epidemiological data are recorded in Table 1, while data on each species are available in Table 2.

Reports of carcass encounter, injuries and marks that suggested human or fishing interaction were observed in 24 carcasses $(46.1 \%$; 24/52): 13 I. geoffrensis $(54.2 \% ; 13 / 24)$ and $11 \mathrm{~S}$. fluviatilis $(39.3 \%$; $11 / 28)$. Among these, $62.5 \%(15 / 24)$ were male and $37.5 \%(9 / 24)$ were female, representing 13 adults $(54.2 \%), 10$ juveniles $(41.7 \%)$ and one neonate $(4.2 \%)$.

\section{Pathological findings}

Gross and/or microscopic lesions were observed in $84.6 \%(44 / 52)$ of lung samples examined. Histopathological analysis was precluded in $6(10.3 \%)$ animals due to advanced autolysis. Detailed pathological descriptions are recorded in Table 3. Pneumonia was the most common respiratory disease process $(53.8 \% ; 28 / 52)$. The main etiological diagnoses were verminous pneumonia $(25 \% ; 13 / 52)$, bacterial pneumonia $(25 \% ; 13 / 52)$ and a single case of meconium aspiration syndrome $(1.9 \%)$. An etiology was not determined in $36.5 \%$ (19/52) or animals (see 'Miscellaneous', below). These pathological categories are described below.

Verminous pneumonia. Pulmonary nematodiasis affected 6/24 (25\%) I. geoffrensis and 7/28 (25\%) S. fluviatilis, involving $26.9 \%$ of sampled adults and $26.6 \%$ of juveniles, both sexes indiscriminantly, and $20 \%$ of neonates. Grossly, verminous pneumonias were characterized by multifocal, poorly demarcated, pale pink/yellow to dark grey, firm foci within the pulmonary parenchyma, typically associated with secondary bronchi and bronchioles (Fig. 2A). Microscopically, the main findings included eosinophilic and suppurative inflammatory exudate admixed with fibrin and karyorhectic debris in early lesions or those foci where larvae predominated (Fig. 2D). With chronicity and/or presence of adult forms this inflammatory exudate became more pyogranulomatous, to include reactive macrophages and multinucleated giant cells (foreign body type), and the alveolar septa and 
Table 1. Carcass discovery date, sex, total body length (TBL), age class, carcass condition, nutritional status and decomposition code of Amazon river dolphins Inia geoffrensis and tucuxis Sotalia fluviatilis from the mid-Solimões River, Amazon, Brazil (1995-2016). M: male; F: female; nd: not determined; J: juvenile; A: adult; N: neonate; EHFI: evidence of human or fishery interaction; Fr: fresh; MA: moderate autolysis; AA: advanced autolysis

\begin{tabular}{|c|c|c|c|c|c|c|c|}
\hline $\begin{array}{l}\text { Case } \\
\text { no. }\end{array}$ & $\begin{array}{l}\text { Discovery } \\
\text { date }\end{array}$ & Sex & $\begin{array}{l}\text { TBL } \\
(\mathrm{m})\end{array}$ & $\begin{array}{l}\text { Age } \\
\text { class }\end{array}$ & Carcass condition and gross observations & $\begin{array}{l}\text { Nutritional } \\
\text { status }\end{array}$ & $\begin{array}{l}\text { Decomposition } \\
\text { state }\end{array}$ \\
\hline \multicolumn{8}{|c|}{ Inia geoffrensis } \\
\hline 1 & 13-Oct-95 & M & 1.34 & $\mathrm{~J}$ & EHFI & nd & nd \\
\hline 2 & 07-Jan-96 & $\mathrm{F}$ & 1.82 & $\mathrm{~A}$ & EHFI & nd & $\mathrm{Fr}$ \\
\hline 3 & 05-Jan-97 & M & 1.45 & $\mathrm{~J}$ & EHFI & nd & $\mathrm{Fr}$ \\
\hline 4 & 24-Jan-01 & $\mathrm{M}$ & 2.18 & A & EHFI: Injured with machete & nd & nd \\
\hline 5 & 26-Nov-05 & $\mathrm{F}$ & 1.73 & $\mathrm{~A}$ & EHFI: Injured with machete & nd & nd \\
\hline 6 & 20-Jul-07 & $\mathrm{F}$ & 1.48 & $\mathrm{~J}$ & nd & nd & MA \\
\hline 7 & 28-Aug-07 & $\mathrm{F}$ & 1.45 & $\mathrm{~J}$ & EHFI & nd & Fr \\
\hline 8 & 14-Jan-08 & $\mathrm{M}$ & 2.08 & A & EHFI: Captured in fishing net and killed & Good & $\mathrm{Fr}$ \\
\hline 9 & 08-Oct-10 & $\mathrm{F}$ & 2.02 & A & nd & Good & $\mathrm{Fr}$ \\
\hline 10 & 31-May-11 & $\mathrm{M}$ & 1.9 & $\mathrm{~J}$ & EHFI: Harpooned in thorax with lung involvement & nd & MA \\
\hline 11 & 25-Aug-11 & nd & 1.26 & $\mathrm{~J}$ & nd & Good & MA \\
\hline 12 & 10-Nov-11 & nd & 1.98 & A & nd & Emaciated & $\mathrm{AA}$ \\
\hline 13 & 30-Nov-11 & nd & 1.93 & A & Used as bait & nd & $\mathrm{Fr}$ \\
\hline 14 & 08-May-12 & $\mathrm{M}$ & 1.87 & A & Trapped inside a flooded floating house & Good & Fr \\
\hline 15 & 14-May-12 & M & 1.22 & $\mathrm{~J}$ & EHFI & nd & Fr \\
\hline 16 & 31-Aug-12 & $\mathrm{F}$ & 1.5 & $\mathrm{~J}$ & EHFI: Possible cranial trauma & nd & nd \\
\hline 17 & 08-Dec-12 & $\mathrm{F}$ & 0.85 & $\mathrm{~N}$ & nd & Emaciated & $\mathrm{AA}$ \\
\hline 18 & 19-Feb-13 & M & 2.13 & A & EHFI: Multiple harpoon and machete wounds & nd & MA \\
\hline 19 & 29-Nov-13 & $\mathrm{F}$ & 1.39 & $\mathrm{~J}$ & $\begin{array}{l}\text { EHFI: Fishing net marks and bruises on the skin; } \\
\text { Necrotic wound on pectoral flipper }\end{array}$ & nd & MA \\
\hline 20 & 17-Jan-14 & M & 2.38 & $\mathrm{~A}$ & EHFI: Perforating wound in right thorax & nd & Fr \\
\hline 21 & 30-Jan-14 & $\mathrm{F}$ & 2.04 & A & nd & nd & MA \\
\hline 22 & 01-Jun-16 & $\mathrm{F}$ & 1.45 & $\mathrm{~J}$ & nd & Good & Fr \\
\hline 23 & 08-Jul-16 & nd & 1.9 & $\mathrm{~A}$ & nd & nd & $\mathrm{AA}$ \\
\hline 24 & 17-Oct-16 & M & 2.01 & A & nd & Good & $\mathrm{AA}$ \\
\hline \multicolumn{8}{|c|}{ Sotalia fluviatilis } \\
\hline 25 & 16-Jun-95 & nd & 0.82 & $\mathrm{~N}$ & nd & nd & MA \\
\hline 26 & 13-Oct-95 & $\mathrm{M}$ & 1.46 & A & EHFI & nd & MA \\
\hline 27 & $08-$-Oct-97 & nd & nd & nd & nd & nd & nd \\
\hline 28 & 17-Jul-98 & M & 0.73 & $\mathrm{~N}$ & $\begin{array}{l}\text { Trapped under floats; rescued and died on } \\
\text { second day of rehabilitation }\end{array}$ & nd & $\mathrm{Fr}$ \\
\hline 29 & 03-Nov-98 & $\mathrm{F}$ & 1.13 & $\mathrm{~J}$ & nd & nd & $\mathrm{AA}$ \\
\hline 30 & 25-Aug-01 & $\mathrm{F}$ & 0.84 & $\mathrm{~J}$ & EHFI & nd & Fr \\
\hline 31 & 25-Aug-05 & M & 1.52 & A & EHFI & nd & nd \\
\hline 32 & 25-Aug-05 & $\mathrm{F}$ & 1.46 & $\mathrm{~A}$ & EHFI & nd & nd \\
\hline 33 & 25-Aug-05 & M & 1.52 & A & EHFI: harpooned and injured with machete & nd & $\mathrm{AA}$ \\
\hline 34 & 07-Nov-05 & $\mathrm{F}$ & 1.39 & A & nd & nd & nd \\
\hline 35 & 03-Dec-05 & $\mathrm{F}$ & 1.24 & A & $\begin{array}{c}\text { Captured alive with respiratory disorder; } \\
\text { died during transport }\end{array}$ & nd & Fr \\
\hline 36 & 23-Oct-06 & M & 1.17 & A & EHFI & nd & nd \\
\hline 37 & 03-Dec-07 & $\mathrm{F}$ & 1.40 & A & nd & nd & MA \\
\hline 38 & $04-$-Oct-10 & $\mathrm{F}$ & 1.30 & A & EHFI: cranial trauma and cuts on flippers & Good & MA \\
\hline 39 & 11-Oct-10 & nd & nd & nd & nd & nd & nd \\
\hline 40 & $13-$ Oct-10 & $\mathrm{M}$ & 1.45 & A & nd & nd & MA \\
\hline 41 & 28 -Aug-11 & $\mathrm{M}$ & nd & nd & nd & nd & nd \\
\hline 42 & $11-$ Sep-11 & $\mathrm{M}$ & 0.92 & $\mathrm{~N}$ & nd & nd & MA \\
\hline 43 & 12-Sep-11 & $\mathrm{F}$ & 1.54 & A & nd & nd & Fr \\
\hline 44 & $17-F e b-12$ & nd & nd & nd & nd & nd & nd \\
\hline 45 & $18-F e b-12$ & $\mathrm{M}$ & 1.4 & $\mathrm{~A}$ & EHFI: large abdominal penetrating incision & nd & MA \\
\hline 46 & 20-May-12 & M & 1.21 & $\mathrm{~J}$ & EHFI & Good & Fr \\
\hline 47 & 20-May-12 & M & 1.32 & $\mathrm{~J}$ & EHFI & nd & Fr \\
\hline 48 & 06-Jun-12 & $\mathrm{F}$ & 0.99 & $\mathrm{~N}$ & EHFI & nd & $\mathrm{Fr}$ \\
\hline 49 & 06-Jun-12 & $\mathrm{F}$ & nd & nd & nd & nd & nd \\
\hline 50 & 07 -Aug-12 & $\mathrm{M}$ & 1.6 & A & nd & nd & nd \\
\hline 51 & $01-D e c-12$ & $\mathrm{M}$ & 1.38 & $\mathrm{~J}$ & nd & nd & nd \\
\hline 52 & 17-Jan-14 & nd & nd & nd & nd & nd & nd \\
\hline
\end{tabular}


Table 2. Summary of sex, age class and decomposition code of Amazon river dolphins Inia geoffrensis and tucuxis Sotalia fluviatilis from the mid-Solimões River, Amazon, Brazil (1995-2016), by species. Abbreviations as in Table 1

\begin{tabular}{|c|c|c|c|c|c|c|c|c|c|c|c|c|}
\hline \multirow[t]{2}{*}{ Species } & \multicolumn{3}{|c|}{$\longrightarrow$ Sex $\longrightarrow$} & \multicolumn{4}{|c|}{$\longrightarrow$ Age class } & \multicolumn{4}{|c|}{ — Decomposition state - } & \multirow[t]{2}{*}{ Total } \\
\hline & Male & Female & nd & Neonate & Juvenile & Adult & nd & Fr & MA & AA & nd & \\
\hline Inia geoffrensis & 10 & 11 & 3 & 1 & 11 & 13 & 0 & 10 & 6 & 4 & 4 & 24 \\
\hline Sotalia fluviatilis & 12 & 11 & 5 & 4 & 5 & 13 & 5 & 7 & 7 & 2 & 12 & 28 \\
\hline Total & 22 & 22 & 8 & 5 & 16 & 26 & 5 & 17 & 13 & 6 & 16 & 52 \\
\hline
\end{tabular}

interstitium became infiltrated by lymphocytes, plasma cells and macrophages (Fig. 2D). More chronic lesions were characterized by well-demarcated, sclerotic granulomas and varying degrees of parenchymal remodeling wherein fibrosis predominated and occasional degenerating nematode debris could be found. All of these cases presented intra-alveolar, -bronchiolar or -bronchial adult nematodes (as suggested by the presence of developed gonads, typically containing viviparous larvae) (Fig. 2D), and immature nematode stages were only observed in 3 cases. In $84.6 \%$ (11/13) of cases, parasites were morphologically compatible with Halocercus brasiliensis (Fig. 2D). Case 35, an adult female $S$. fluviatilis, was sighted alive with tachypnea, oozing white foam through the blowhole, and was unable to dive. The animal was captured and taken to a rehabilitation facility; however, it died during transport.

Bacterial pneumonia. Bacterial pneumonia, chiefly represented as suppurative bronchopneumonia, was diagnosed in $33.35 \%(8 / 24)$ of $I$. geoffrensis and $17.6 \%(5 / 28)$ of $S$. fluviatilis, involving $46.1 \%(6 / 13)$ of sampled males and $38.5 \%(5 / 13)$ of females. Grossly, bronchi and bronchioles were expanded by varying amounts of yellow to pink exudate that extended into and distorted the adjacent parenchyma. Microscopically, variably distended bronchi and bronchioles (bronchiectasis) and alveoli contained abundant viable and degenerate neutrophils admixed with fibrin, necrotic cellular debris and edema. In these cases, varying numbers of bacteria, including presumably single or multiple kinds of Gram-positive and Gram-negative coccoid and bacillary bacteria, respectively, were noted within the inflammatory exudate. With chronicity, the alveolar septa and bronchial/bronchiolar submucosa had mild lymphoplasmacytic infiltrates and remodeling phenomena, mainly represented by fibrosis, distorting the parenchyma and distal bronchioles. In case 20, there was right pleural empyema (pyothorax) and severe fibrinosuppurative pleuritis of the right lung (Fig. 2B,C), associated with a penetrating wound on the right thorax. Microscopically, there was severe, diffuse, chronic fibrinosuppurative pleuropneumonia with myriad Gram-positive cocci (Fig. 2E) and granulation tissue. Coinfection by mixed Gram-positive and Gram-negative bacteria and $H$. brasiliensis was histologically confirmed in case 48.

Meconium aspiration syndrome. A neonate $S$. fluviatilis (case 25) presented marked, diffuse pulmonary atelectasia with abundant aspirated intrabronchial, -bronchiolar and -alveolar individual squames or most often large aggregates of keratinized stratified epithelium with retained nuclei (pulmonary vernix caseosa), along with brown granular particles or aggregates, interpreted as meconium (Fig. 2F).

Miscellaneous. In 19 animals, gross and/or microscopic lesions could not be ascribed to a defined etiology (I. geoffrensis, 8/24; S. fluviatilis, 11/28). These cases included minimal to mild non-specific microscopic findings such as alveolar histiocytosis, atelectasia, emphysema, interstitial fibrosis and bronchial/ bronchiolar mineralization. Nonetheless, 5 of them had mild multifocal foci of chronic lymphoplasmacytic and histiocytic interstitial to bronchointerstitial pneumonia that, together with focal granuloma, fibrosis and angiomatosis, could suggest nematodal parasitization. Abundant aspirated mineral material (suggestive of sediment) or keratin squames, associated with edema and hemorrhage, was observed in 1 I. geoffrensis and 3 S. fluviatilis $(7.8 \% ; 4 / 52)$.

\section{DISCUSSION}

Cetaceans have been recognized as suitable bioindicators to monitor the health of aquatic ecosystems (Bossart 2011). Pulmonary infectious disease is a leading cause of morbidity in free-ranging and captive cetaceans, often leading to stranding and/or death (Dunn \& Buck 2001, Venn-Watson et al. 2012). In this regard, health and disease aspects of free-ranging populations of Inia geoffrensis and Sotalia fluviatilis remain largely unknown. Pathology-based and pref- 
Table 3. Pulmonary gross and microscopic lesions, and etiological diagnoses in Amazon river dolphins Inia geoffrensis and tucuxis Sotalia Ofluviatilis from the mid-Solimões River, Amazon, Brazil (1995-2016). Case nos. as in Table 1. AA: advanced autolysis; NSLO: no significant lesions observed; NLR: no lesions reported; nd: not determined

\begin{tabular}{|c|c|c|c|}
\hline $\begin{array}{l}\text { Case } \\
\text { no. }\end{array}$ & $\begin{array}{l}\text { Gross } \\
\text { findings }\end{array}$ & Microscopic findings & $\begin{array}{l}\text { Etiological } \\
\text { diagnosis }\end{array}$ \\
\hline 1 & NLR & Minimal, scattered mononuclear interstitial infiltrates & nd \\
\hline 2 & NLR & Minimal, scattered mononuclear interstitial infiltrates and alveolar histiocytosis & nd \\
\hline 3 & NLR & Marked, focally extensive, subacute fibrinosuppurative bronchopneumonia & $\begin{array}{c}\text { Bacterial } \\
\text { pneumonia }\end{array}$ \\
\hline 4 & NLR & $\begin{array}{l}\text { Mild, multifocal edema with rare alveolar histiocytosis } \\
\text { and lymphohistiocytic interstitial infiltrates }\end{array}$ & nd \\
\hline 5 & NLR & Moderate, multifocal, acute hemorrhage & nd \\
\hline 6 & $\begin{array}{l}\text { Scattered, pale tan to } \\
\text { dark nodules within } \\
\text { parenchyma bilaterally }\end{array}$ & $\begin{array}{l}\text { Mild to marked, multifocal, chronic lymphoplasmacytic broncho- } \\
\text { interstitial pneumonia with interstitial and subpleural fibrosis, } \\
\text { hemorrhage and aspirated bacteria; mild subpleural emphysema }\end{array}$ & nd \\
\hline 7 & NSLO & $\begin{array}{c}\text { Mild, focal lymphohistiocytic bronchitis with intralesional nematode } \\
\text { debris; congestion and mild edema }\end{array}$ & $\begin{array}{l}\text { Verminous } \\
\text { pneumonia }\end{array}$ \\
\hline 8 & NSLO & $\begin{array}{l}\text { Mild, multifocal, chronic lymphoplasmacytic bronchointerstitial pneumonia with } \\
\text { ld edema; rare aspirated mineral material; multifocal atelectasia and emphysema }\end{array}$ & nd \\
\hline 9 & NSLO & Mild edema with abundant aspirated mineral material & nd \\
\hline 10 & $\begin{array}{l}\text { Lung perforation } \\
\text { by harpoon }\end{array}$ & Marked, focally extensive suppurative bronchopneumonia & $\begin{array}{c}\text { Bacterial } \\
\text { pneumonia }\end{array}$ \\
\hline 11 & NSLO & AA & nd \\
\hline 12 & NLR & $\begin{array}{l}\text { Marked, multifocal, subacute fibrinosuppurative and necrotizing } \\
\text { bronchopneumonia with vasculitis, occlusive thrombosis and hemorrhage }\end{array}$ & $\begin{array}{c}\text { Bacterial } \\
\text { pneumonia }\end{array}$ \\
\hline 13 & NLR & $\begin{array}{c}\text { Moderate, focally extensive, subacute pyogranulomatous bronchopneumonia } \\
\text { with nematodes (Halocercus brasiliensis) }\end{array}$ & $\begin{array}{l}\text { Verminous } \\
\text { pneumonia }\end{array}$ \\
\hline 14 & $\begin{array}{l}\text { Edema and congestion; } \\
\text { scattered, pale tan } \\
\text { to dark nodules within } \\
\text { parenchyma bilaterally }\end{array}$ & $\begin{array}{c}\text { Moderate, multifocal, chronic pyogranulomatous } \\
\text { bronchitis with nematodes }(H . \text { brasiliensis })\end{array}$ & $\begin{array}{l}\text { Verminous } \\
\text { pneumonia }\end{array}$ \\
\hline 15 & NLR & Mild, multifocal, chronic mononuclear interstitial pneumonia & nd \\
\hline 16 & NSLO & $\begin{array}{c}\text { Mild, multifocal, chronic lymphohistiocytic interstitial pneumonia } \\
\text { with nematodes (H. brasiliensis) and edema }\end{array}$ & $\begin{array}{l}\text { Verminous } \\
\text { pneumonia }\end{array}$ \\
\hline 17 & NSLO & $\begin{array}{l}\text { Mild, focal, subacute suppurative bronchopneumonia; mild, multifocal } \\
\text { intra-alveolar keratin squames with rare brown aspirated material }\end{array}$ & $\begin{array}{l}\text { Bacterial } \\
\text { pneumonia }\end{array}$ \\
\hline 18 & NSLO & $\begin{array}{l}\text { Mild, multifocal, chronic lymphohistiocytic interstitial pneumonia and } \\
\text { pleuritis with nematode debris and fibrosis; congestion and scattered } \\
\text { alveolar hemorrhage; mild, multifocal angiomatosis; multifocal atelectasia }\end{array}$ & $\begin{array}{l}\text { Verminous } \\
\text { pneumonia }\end{array}$ \\
\hline 19 & $\begin{array}{l}\text { Scattered, pale tan to } \\
\text { dark nodules within paren- } \\
\text { chyma bilaterally; edema } \\
\text { and congestion }\end{array}$ & AA & nd \\
\hline 20 & $\begin{array}{l}\text { Pyothorax associated with } \\
\text { right thoracic perforating } \\
\text { wound and severe } \\
\text { fibrinosuppurative pleuritis }\end{array}$ & $\begin{array}{l}\text { Severe, diffuse, chronic fibrinosuppurative pleuritis } \\
\text { and bronchopneumonia with numerous Gram-positive cocci }\end{array}$ & $\begin{array}{c}\text { Bacterial } \\
\text { pneumonia }\end{array}$ \\
\hline 21 & $\begin{array}{l}\text { Hydrothorax; pulmonary } \\
\text { edema and congestion; } \\
\text { scattered pale tan to yellow } \\
\text { nodules within parenchyma } \\
\text { laterally; focal left lung abscess }\end{array}$ & $\begin{array}{l}\text { Mild, focal suppurative bronchopneumonia with focal } \\
\text { interstitial fibrosis }\end{array}$ & $\begin{array}{c}\text { Bacterial } \\
\text { pneumonia }\end{array}$ \\
\hline 22 & $\begin{array}{l}\text { Scattered, pale tan } \\
\text { to dark nodules within } \\
\text { parenchyma bilaterally; } \\
\text { edema }\end{array}$ & $\begin{array}{l}\text { Moderate, diffuse, chronic lymphoplasmacytic } \\
\text { bronchointerstitial pneumonia with mucopurulent bronchitis }\end{array}$ & $\begin{array}{c}\text { Bacterial } \\
\text { pneumonia }\end{array}$ \\
\hline 23 & $\begin{array}{l}\text { Hydrothorax; focal parasitic } \\
\text { granuloma in right lung }\end{array}$ & AA; mild, multifocal intrabronchial nematodes (H. brasiliensis) & $\begin{array}{l}\text { Verminous } \\
\text { pneumonia }\end{array}$ \\
\hline 24 & AA & AA & nd \\
\hline 25 & NSLO & $\begin{array}{l}\text { Marked, diffuse atelectasia with marked aspiration } \\
\text { of keratin squames and meconium plugs }\end{array}$ & $\begin{array}{l}\text { Meconium } \\
\text { aspiration } \\
\text { syndrome }\end{array}$ \\
\hline 26 & NSLO & AA & nd \\
\hline
\end{tabular}


Table 3 (continued)

\begin{tabular}{lc}
$\begin{array}{l}\text { Case } \\
\text { no. }\end{array}$ & $\begin{array}{c}\text { Gross } \\
\text { findings }\end{array}$ \\
\hline 27 & NLR \\
28 & NSLO \\
29 & NSLO \\
30 & NSLO \\
31 & NSLO \\
32 & NSLO \\
33 & NSLO \\
34 & NSLO
\end{tabular}

$35 \quad$ Animal found alive presenting tachypnea, pulmonary edema and unable to dive; multiple

pale tan to yellow

nodules within parenchyma bilaterally

NLR

NSLO

NLR

NLR

NSLO

Mild, focally extensive suppurative bronchopneumonia with necrosis

Mild, multifocal suppurative bronchopneumonia with necrosis; mild, diffuse edema with scattered keratin squames and scattered hemorrhage

Mild, focal pleural fibrosis; atelectasia; mild, multifocal angiomatosis and congestion Mild edema with scattered alveolar histiocytosis and rare hemorrhage

Mild, multifocal, chronic suppurative bronchointerstitial pneumonia with occasional type II pneumocyte hyperplasia, mild interstitial fibrosis, edema and scattered hemorrhage; mild angiomatosis

Mild, multifocal, chronic interstitial pneumonia and focal granuloma; congestion; mild, multifocal angiomatosis

Mild, multifocal, chronic suppurative bronchointerstitial pneumonia with mild fibrosis, edema and scattered hemorrhage

Moderate, focally extensive, chronic proliferative pleuritis; mild, multifocal, chronic suppurative bronchopneumonia with mild interstitial fibrosis

Moderate to marked, multifocal, chronic pyogranulomatous

bronchopneumonia with nematodes $(H$. brasiliensis), epithelial hyperplasia, mild type II pneumocyte hyperplasia, fibrosis, bronchoconstriction and edema; mild, multifocal arterial tunica media hypertrophy/hyperplasia and angiomatosis

Mild, multifocal, chronic bronchointerstitial pneumonia with scattered neutrophilic exudate, mild edema and rare intra-alveolar aspirated material

Mild, multifocal, chronic interstitial fibrosis with rare mononuclear infiltrates; atelectasia; congestion

Mild edema and congestion

Diffuse atelectasia; mild, multifocal interstitial fibrosis

$$
\begin{aligned}
& \text { AA } \\
& \text { AA }
\end{aligned}
$$

Minimal to mild, scattered mononuclear interstitial infiltrates with edema, alveolar histiocytosis and occasional aspirated squames and aspirated mineral material

Mild, multifocal, chronic bronchointerstitial pneumonia; scattered bronchial/ bronchiolar mineralization

Mild, diffuse edema with rare hemorrhage; multifocal atelectasia; mild arterial tunica media hypertrophy/hyperplasia

Mild to marked, multifocal, chronic pyogranulomatous bronchopneumonia with nematodes ( $H$. brasiliensis), bronchiectasis, bronchitis, bronchial sclerosis, edema and mild bronchoconstriction; scattered bronchial/bronchiolar mineralization

Marked, multifocal to coalescing, chronic pyogranulomatous and

fibrosing bronchopneumonia with numerous nematodes ( $H$. brasiliensis), bronchiectasis, fibrosis, bronchoconstriction, diffuse edema and hemorrhage; scattered bronchial/bronchiolar mineralization; mild arterial tunica media hypertrophy/ hyperplasia and angiomatosis

Moderate, multifocal, chronic bronchointerstitial lymphoplasmacytic and eosinophilic pneumonia with focal granuloma, edema, and mild fibrosis

Marked, multifocal to coalescing, chronic eosinophilic and suppurative to pyogranulomatous and fibrosing bronchointerstitial pneumonia with bronchitis, bronchiectasis, numerous adult nematodes and rare larvae $(H$. brasiliensis) and mixed bacteria, lymphoid follicle formation, bronchoconstriction and edema; arterial tunica media hypertrophy/hyperplasia

Marked, multifocal to coalescing, chronic pyogranulomatous bronchopneumonia with numerous nematodes (H. brasiliensis), necrosis, fibrosis, bronchoconstriction and edema

Mild, multifocal, chronic pyogranulomatous bronchopneumonia with nematodes $(H$. brasiliensis)

Moderate, multifocal, chronic pyogranulomatous bronchopneumonia with nematodes ( $H$. brasiliensis), fibrosis and bronchiectasis

NSLO
Etiological diagnosis

Bacterial pneumonia

Bacterial pneumonia nd nd

Bacterial pneumonia

nd

Bacterial pneumonia

Bacterial pneumonia Verminous pneumonia

Bacterial pneumonia nd

nd

nd

nd

nd

nd

nd

nd

Verminous pneumonia

Verminous pneumonia

nd

Verminous pneumonia (with

bacterial coinfection)

Verminous pneumonia

Verminous pneumonia Verminous pneumonia nd 

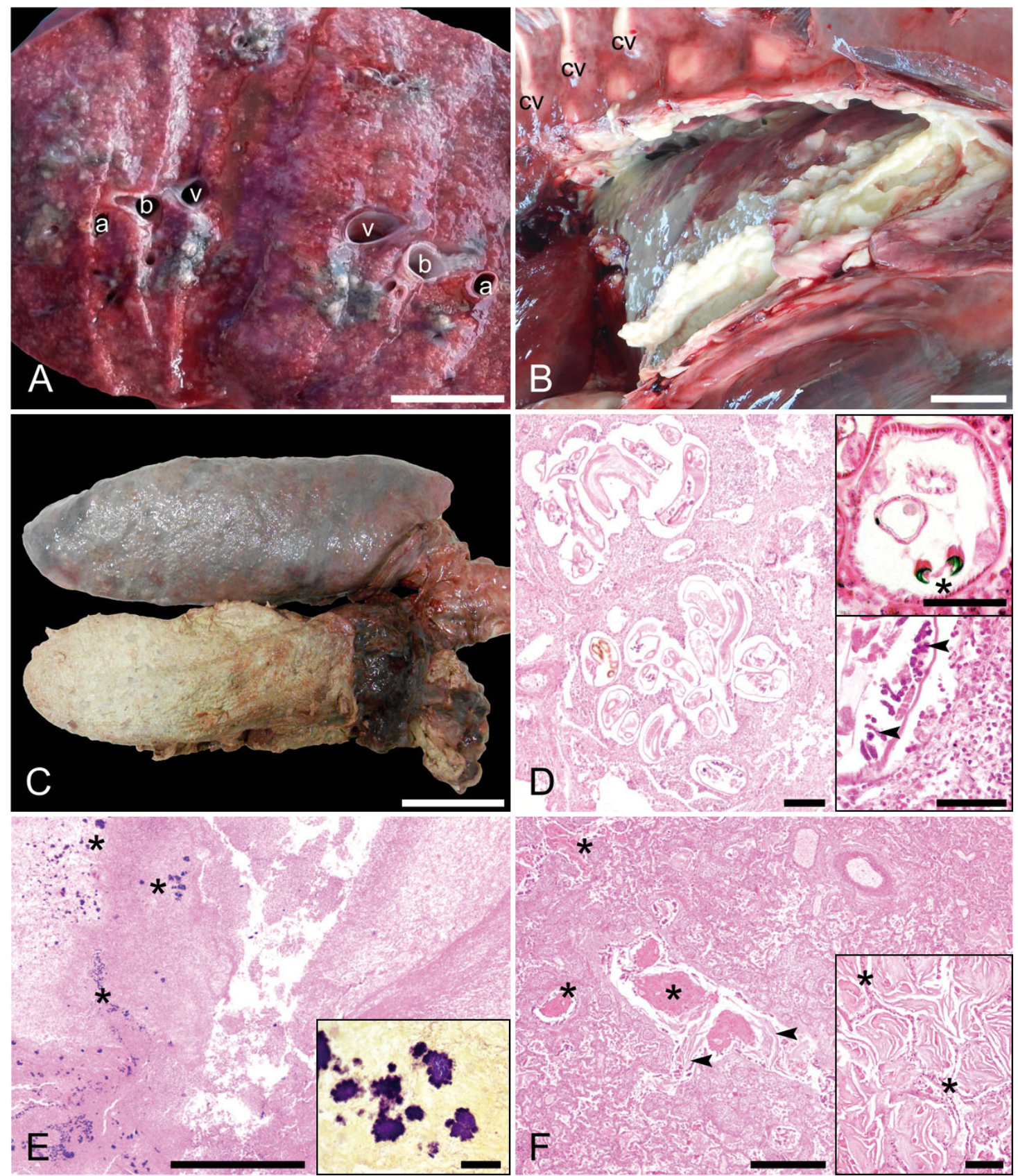

Fig. 2. Macroscopic (A-C) and microscopic (D-F) pulmonary lesions in free-ranging Amazon river dolphins Inia geoffrensis and tucuxis Sotalia fluviatilis from the mid-Solimões River, Amazon, Brazil (1995-2016). Case nos. as in Table 1. (A) Case 48. Multifocal, poorly demarcated, pale tan/grey to dark foci typically associated with secondary bronchi and bronchioles wherein nematodes were confirmed histologically. Scale bar $=2 \mathrm{~cm}$. a, artery; b, bronchus; v, vein. (B) Case 20. Right pleural empyema (pyothorax) and fibrinosuppurative pleuritis. Scale bar $=5 \mathrm{~cm} . \mathrm{Cv}$, costovertebral joints. (C) Case 20. Severe fibrinosuppurative pleuritis of right lung, ex situ. Scale bar $=10 \mathrm{~cm}$. (D) Case 48. Pleocellular (suppurative, eosinophilic and granulomatous) bronchopneumonia with numerous Halocercus brasiliensis nematodes. H\&E staining. Scale bar $=500 \mu \mathrm{m}$. Upper inset: detail of caudal end of male $H$. brasiliensis with spicules (asterisk). H\&E staining. Scale bar $=200 \mu$ m. Lower inset: detail of mid-caudal segment of female $H$. brasiliensis with viviparous larvae (arrowheads). H\&E. Scale bar $=200 \mu m$. (E) Case 20. Severe fibrinosuppurative pleuritis with deeply basophilic bacterial aggregates (asterisks). H\&E staining. Scale bar $=500 \mu$. Inset: Gram-positive cocci within inflammatory exudate. Gram/Twort staining. Scale bar $=20 \mu \mathrm{m}$. (F) Case 25. Diffuse atelectasis with secondary bronchus and branching bronchioles plugged with meconium (asterisks) and keratin squames (arrowheads). H\&E staining. Scale bar $=200 \mu \mathrm{m}$. Inset: the alveoli are expanded by abundant keratin squames and alveolar septa are thinned and displaced (asterisks). Scale bar $=50 \mu \mathrm{m}$ 
erentially long-term studies on these locally endangered species are necessary to provide better understanding of the causes of debilitation and mortality that may play a role in population decline. Additionally, these riverine species may provide valuable insight into aquatic ecosystem health, which also has implications for public health. Post mortem investigations have been used as valuable tools to assess health status on most free-living cetacean species (Arbelo et al. 2013); however, they involve arduous, complex and demanding research and logistics. Despite the fact that these pathologic investigations may have obvious biases and limitations (e.g. examined animals are not fully representative of the live population, advanced decomposition of corpses at necropsy examination), the gain of scientific knowledge and associated outcomes, e.g. policy measures, exceed and justify the troubles. In the present study, we detected a high occurrence of pulmonary disease in I. geoffrensis and S. fluviatilis from mid-Solimões River. However, inherent difficulties (e.g. complicated access to carcasses at remote Amazonian areas, unfavorable climate conditions) precluded an immediate necropsy response and thus limited post mortem evaluations for some animals.

Most cases with pulmonary lesions were attributed to either verminous pneumonia or bacterial pneumonia from which chronic lesion presentations predominated. Although several cases with moderate and marked parasitic and/or bacterial pneumonia were detected in this study, mild lesions predominated. The high prevalence of lung disease in our study was in agreement with previous studies (Measures 2001, Fauquier et al. 2009); however, a clear-cut, definitive contributory role as to cause of death was not readily evident except in a few cases. Verminous pneumonias were mainly associated with Halocercus brasiliensis. The genus Halocercus (Nematoda; Pseudaliidae; Metastrongyloidea) encompasses some of the most common lungworms present in odontocete species (Woodard et al. 1969, Measures 2001). In the present study, all verminous pneumonia cases in which it was possible to identify the nematode were caused by $H$. brasiliensis. This parasite is known to infect the respiratory tract of odontocete cetaceans worldwide (Measures 2001), and reported hosts in Brazil may include striped dolphins Stenella coeruleoalba and Guiana dolphin Sotalia guianensis (Marigo et al. 2010). This nematode affected $88 \%$ of $S$. guianensis from the southern and southeastern coasts of Brazil, causing lung lesions that, in $40 \%$ of these cases, were considered the probable cause of death (Marigo et al. 2010). In the present study, the observed lesions recapitulate histopathological features described in previous studies, ranging from acute inflammatory exudation to remodeling due to mononuclear interstitial inflammation and fibrosis in more chronic stages (Woodard et al. 1969, Fauquier et al. 2009, Marigo et al. 2010). Two of the authors (J.D.D., J.L.C.D.) have observed marked pulmonary arterial tunica media hypertrophy/hyperplasia in free-ranging $S$. guianensis with $H$. brasiliensis infestation, suggesting localized hypertensive phenomena (unpubl. obs.); however, a comparable vascular response was lacking in I. geoffrensis and S. fluviatilis from the present study. In this study, the pathologic impact and contributory role to stranding and/ or death could not be determined. To the best of our knowledge, this is the first report of $H$. brasiliensis in I. geoffrensis. Further studies may focus on host- and parasite-specific features to explain potential diverging pathological responses to this lungworm.

Little is known of the life cycle of any of the pseudaliids (Measures 2001); however, vertical transmission of Halocercus spp. in cetaceans has been suggested (Moser \& Rhinehart 1993). Similarly, we observed pulmonary nematodiasis by $H$. brasiliensis in a female neonate $S$. fluviatilis, further supporting a transplacental infestation and widening the host record where this route of transmission appears to occur.

Bacterial pneumonia has been recorded in various cetaceans, and several species of bacteria have been isolated from lungs, pleura and thoracic cavity (Higgins 2000). Frequently, these isolates tend to be Gram-negative bacteria. In addition, bacterial pleuritis and pneumonia may be primary sources of septic process in dolphins (Arbelo et al. 2013). The predominant pulmonary bacterial isolates reported in captive I. geoffrensis were Proteus sp., Morganella morganii, Alcaligenes sp., Citrobacter sp., Chryseobacterium sp., Escherichia coli, Pasteurella sp., Pseudomonas sp., Pneumococcus sp. and Streptococcus sp. (Bonar et al. 2007). Of particular interest, I. geoffrensis is also known to suffer from infections of Streptococcus iniae ('golf ball disease') (Bonar \& Wagner 2003, Bonar et al. 2007), a Gram-positive coccus of emerging global zoonotic importance that affects fish, cetaceans and humans. In I. geoffrensis, this bacterium has been associated with abscedative dermatitis and panniculitis, which may lead to pyogenic septicemia, affecting various organs, including the lungs (Song et al. 2017). Although we could not perform bacteriological analyses in suspected diseased lungs with suppurative bronchopneumonia and/or pleuropneumonia, we detected Gram-posi- 
tive cocci in some cases. Streptococcus iniae infection was considered a highly probable etiology in these cases. Due to its zoonotic importance and the highly prevalent artisanal fishery in the mid-Solimões River along with fresh fish consumption, a public health concern is raised. Future studies may try to assess presence of this zoonotic bacterium in these areas and human risk exposure.

We observed pathological findings consistent with meconium aspiration syndrome (MAS) in a neonate $S$. fluviatilis. The microscopic features-i.e. abundant aspirated intrabronchial, -bronchiolar and -alveolar individual squames and/or aggregates of keratinized stratified epithelium with retained nuclei (pulmonary vernix caseosa), along with brown granular particles or aggregates - coincide with previous descriptions in humans and other animals, including odontocetes and mysticetes (Tanaka et al. 2014). MAS is a neonatal pathology associated with serious respiratory disorder caused by aspiration of meconium-stained amniotic fluid in the airways during intrauterine gasping or during the first few breaths (Srinivasan \& Vidyasagar 1999). Meconium passage to amniotic fluid is generally considered an indicator of fetal distress and the aspiration occurs in response to fetal anoxia or hypoxia (Alonso-Spilsbury et al. 2005). The present case represents the first description of MAS in S. fluviatilis, and reinforces the consideration of MAS as a differential diagnosis of severe lung disease in odontocete neonatal mortality and stillbirth.

In a set of animals, gross and/or microscopic lesions could not be ascribed to a defined etiology. In these cases, an infectious cause, most likely parasitic but not exclusively, could be associated with chronic mononuclear interstitial and bronchointerstitial inflammatory infiltrates. In some cases, abundant aspirated mineral material variably associated with edema and hemorrhage was highly suggestive of sediment aspiration during perimortem agony regardless of the primary cause of death.

Evidence of anthropogenic interactions, compatible with fishing activities, was observed in a remarkable number of carcasses, confirming reports on the high incidence of these interactions in the midSolimões River (Iriarte \& Marmontel 2013b). For some of these human-caused injuries, there was a clear-cut link with lung disease. For instance, in 2 male I. geoffrensis (cases 10 and 20) there were penetrating wounds on the thorax, probably caused by harpoon, which could represent the entry route for pathogenic bacteria causing suppurative or fibrinosuppurative bronchopneumonia.
In summary, these results indicate a high occurrence of pulmonary pathology in free-ranging I. geoffrensis and S. fluviatilis, mainly represented by verminous pneumonia and bacterial pneumonia. For some cases, there was an evident direct link between anthropogenic activities and lung disease. This is the first report of $H$. brasiliensis in I. geoffrensis and the first description of meconium aspiration syndrome in a neonate $S$. fluviatilis. Further research is needed to better delineate the impact of lung disease in these riverine species. Our findings may provide a scientific basis for future medical and conservation efforts on these Amazonian dolphins.

Acknowledgements. This research was funded by the Brazilian National Council for Scientific and Technological Development (CNPq), the Brazilian Federal Agency for Support and Evaluation of Graduate Education (CAPES which provided a doctoral scholarship to T.C.S.R.), the Mamirauá Institute for Sustainable Development (IDSM) and the University of São Paulo. The authors thank the support offered by all past and current staff members of the IDSM's research team on Amazonian aquatic mammals (Mamaq), Luzivaldo Castro dos Santos Júnior, Márcio Sabbadini Francisco and the members of the University of São Paulo's Laboratory of Wildlife Comparative Pathology. J.D.D. is the recipient of a post-doctoral fellowship by the São Paulo Research Foundation (FAPESP; grant 2017/ 02223-8). J.L.C.D. is the recipient of research fellowship from the CNPq (grant 305349/2015-5).

\section{LITERATURE CITED}

Alonso-Spilsbury M, Mota-Rojas D, Villanueva-García D, Martínez-Burnes J and others (2005) Perinatal asphyxia pathophysiology in pig and human: A review. Anim Reprod Sci 90:1-30

Anderson CR, Chabaud AG, Willmott S (2009) Keys to the nematode parasites of vertebrates. Archival volume. CAB International, Wallingford, UK

Arbelo M, Monteros AE, Herráez P, Andrada M and others (2013) Pathology and causes of death of stranded cetaceans in the Canary Islands (1999-2005). Dis Aquat Org 103:87-99

Best RC, da Silva VMF (1984) Preliminary analysis of reproductive parameters of the boutu (Inia geoffrensis) and the tucuxi (Sotalia fluviatilis), in the Amazon River system. In: Perrin WF, DeMaster D, Brownell RJ Jr (eds) Reproduction of Cetacea: with special reference to stock assessment. Int Whaling Commun Spec Pub 6:361-369

Bonar CJ, Wagner RA (2003) A third report of 'golf ball disease' in an Amazon River dolphin (Inia geoffrensis) associated with Streptococcus iniae. J Zoo Wildl Med 34: 296-301

*Bonar CJ, Boede EO, Hartmann MG, Lowenstein-Whaley J and others (2007) Retrospective study of pathologic findings in the Amazon and Orinoco river dolphin (Inia geoffrensis) in captivity. J Zoo Wildl Med 38:177-191

Bossart GD (2011) Marine mammals as sentinel species for oceans and human health. Vet Pathol 48:676-690 
Brazil (2014) Portaria no. 444, de 17 de dezembro de 2014. Diário Oficial da União, Imprensa Nacional 1808, Brasília, DF, 245:121-126

Delyamure SL (1955) Helminthofauna of marine mammals: ecology and phylogeny [Gelmintofauna morskikh mlekopitayushchikh v svete ikh ekologii i filogenii]. Israel Program for Scientific Translations, Jerusalem

Dunn JL, Buck JD (2001) Bacterial diseases. In: Dierauf LA, Gulland FMD (eds) CRC Handbook of marine mammal medicine, 2nd edn. CRC Press, Boca Raton, FL, p 302-336

Fauquier DA, Kinsel MJ, Dailey MD, Sutton GE, Stolen MK, Wells RS, Gulland FMD (2009) Prevalence and pathology of lungworm infection in bottlenose dolphins Tursiops truncatus from southwest Florida. Dis Aquat Org 88: 85-90

Higgins R (2000) Bacteria and fungi of marine mammals: a review. Can Vet J 41:105-116

ICMBio (Instituto Chico Mendes de Conservação da Biodiversidade) (2011) Plano de ação nacional para a conservação dos mamíferos aquáticos - Pequenos cetáceos. ICMBio, Brasília, DF

Iriarte V, Marmontel M (2013a) Insights on the use of dolphins (boto, Inia geoffrensis and tucuxi, Sotalia fluviatilis) for bait in the piracatinga (Calophysus macropterus) fishery in the western Brazilian Amazon. J Cetacean Res Manag 13:163-173

Iriarte V, Marmontel M (2013b) River dolphin (Inia geoffrensis, Sotalia fluviatilis) mortality events attributed to artisanal fisheries in the western Brazilian Amazon. Aquat Mamm 39:116-124

Marigo J, Ruoppolo V, Rosas FCW, Valente ALS, Oliveira MR, Dias RA, Catão-Dias JL (2010) Helminths of Sotalia guianensis (Cetacea: Delphinidae) from the south and southeastern coasts of Brazil. J Wildl Dis 46:599-602

Measures LN (2001) Lungworms of marine mammals. In: Samuel WM, Pybus J, Kocan AA (eds) Parasitic diseases

Editorial responsibility: Stephen Raverty,

Abbotsford, British Columbia, Canada of wild animals, 2nd edn. Iowa State University Press, Ames, IA, p 279-300

Moser M, Rhinehart H (1993) The lungworm, Halocercus spp. (Nematoda: Pseudaliidae) in cetaceans from California. J Wildl Dis 29:507-508

Keeves RR, Jefferson TA, Karczmarski L, Laidre K and others (2011) Inia geoffrensis. The IUCN Red List of Threatened Species. http://dx.doi.org/10.2305/IUCN.UK.20111.RLTS.T10831A3220342.en. Accessed 10 Aug 2017

Secchi E (2012) Sotalia fluviatilis. The IUCN Red List of Threatened Species. http://dx.doi.org/10.2305/IUCN. UK.2012.RLTS.T190871A17583369.en. Accessed 10 Aug 2017

Song Z, Yuel R, Sun Y, Liu C and others (2017) Fatal bacterial septicemia in a bottlenose dolphin Tursiops truncatus caused by Streptococcus iniae. Dis Aquat Org 122: 195-203

Srinivasan HB, Vidyasagar D (1999) Meconium aspiration syndrome: current concepts and management. Compr Ther 25:82-89

* Tanaka M, Izawa T, Kuwamura M, Ozaki M, Nakao T, Ito S, Yamate J (2014) A case of meconium aspiration syndrome in a bottlenose dolphin (Tursiops truncatus) calf. J Vet Med Sci 76:81-84

*Venn-Watson S, Daniels R, Smith C (2012) Thirty year retrospective evaluation of pneumonia in a bottlenose dolphin Tursiops truncatus population. Dis Aquat Org 99: 237-242

Vianna JA, Hollatz C, Marmontel M, Redondo RAF, Santos FR (2010) Amazon river dolphin: high phylopatry due to restricted dispersion at large and short distances. In: Ruiz-Garcia M, Shostell JM (eds) Biology, evolution and conservation of river dolphins within South America and Asia. Nova Publishers, Uniontown, PA, p 101-116

* Woodard JC, Zam SG, Caldwell DK, Caldwell MC (1969) Some parasitic diseases of dolphins. Pathol Vet 6: $257-272$

Submitted: December 18, 2017; Accepted: August 9, 2018 Proofs received from author(s): September 25, 2018 